\section{Auch bei Overlap-Syndrom wirkt CPAP lebensverlängernd}

Machado MCL et al. CPAP and survival in moderate-to-severe obstructive sleep

apnoea syndrome and hypoxaemic COPD. Eur Respir J. 2010;35:132-137

\section{Hintergrund und Fragestellung}

Die chronisch obstruktive Lungenerkrankung (COPD) ist häufig mit Diabetes mellitus und kardiovaskulären Erkrankungen assoziiert. Das Mortalitätsrisiko wird zusätzlich erhöht, wenn die COPD mit einem obstruktiven Schlafapnoesyndrom (OSAS) kombiniert ist (OverlapSyndrom). Dies gilt insbesondere für Patienten, die auch tagsüber eine Hypoxämie aufweisen.

Bis jetzt war unbekannt, wie sich die kontinuierliche positive Atemwegsdruck-Therapie (CPAP) auf die Lebenserwartung von Patienten mit COPD und ausgeprägter Hypoxämie auswirkt.

\section{Methodik}

In einem prospektiven Ansatz wurden 95 Patienten eingeschlossen, die eine COPD und ein mittel- bis schwergradiges OSAS mit einem Apopnoe-Hypopnoe-Index (AHI) von $>15 /$ h hatten und eine Langzeit-Sauerstofftherapie erhielten. Die Sauerstofftherapie erfolgte über eine Nasenbrille.

61 Patienten akzeptierten die CPAPTherapie und führten diese regelmäßig durch. Während der CPAP-Titration wurde die Sauerstoff-Flussrate so gewählt, dass eine arterielle Sauerstoffsättigung $>90 \%$ erreicht wurde. Der mittlere CPAP-Wert betrug 9,8 $\mathrm{cmH}_{2} \mathrm{O}$.

Nur Patienten, die ihre Therapie mehr als fünf Stunden pro Nacht und mehr als fünfTage pro Woche durchführten, wurden in die Auswertung aufgenommen. Als Kontrollgruppe dienten 34 Patienten, die entweder kein CPAP-Gerät erhielten oder dieses ablehnten.

Alle Patienten wurden im Zeitraum von 1996 bis 2006 alle drei Monate kontrolliert. Im Todesfall wurde die Ursache eruiert. Die mittlere Beobachtungszeit betrug 41 Monate (6-106 Monate).

\section{Ergebnisse}

Die Einsekundenkapazität $\left(\mathrm{FEV}_{1}\right)$ betrug in der CPAP-Gruppe 43,4 (\% des Sollwerts) und in der Gruppe ohne CPAP 37,9. Der AHI lag bei 43,2/h bzw. 43,3/h.

Die Gesamtmortalität lag bei $41 \%$. In der nicht mit CPAP behandelten Gruppe traten 27 Todesfälle auf (Mortalität: 79,4\%), in der mit CPAP behandelten Gruppe zwölf (Mortalität: 19,7\%).

Eine Kaplan-Meier-Analyse ergab eine signifikant höhere 5-Jahres-Überlebensrate für die Patienten aus der CPAPGruppe (71 vs. 26\%). Die Hazard Ratio für Tod lag bei 0,19 für Patienten aus der CPAP-Gruppe im Vergleich zur Kontrollgruppe.

\section{Kommentar}

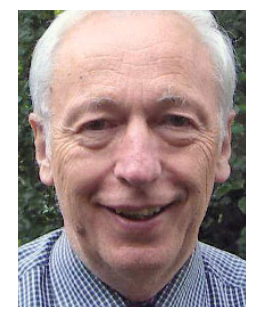

Prof. Dr. med. Karl-Heinz Rühle, Hagen

Die Langzeit-Sauerstofftherapie führt per se zu einer erheblichen Verlängerung der Lebenserwartung gegenüber einer Therapie, die keine Sauerstoffbehandlung beinhaltet $[1,2]$. Die kombinierten Daten der MRC- und der NOTT-Studie ergaben unter einer Langzeit-Sauerstofftherapie eine 5-Jahres-Überlebensrate von 62 vs. 16\%. In der vorliegenden Studie erhöhte die CPAP-Therapie die Überlebensrate von 26 auf $71 \%$.

Die Patienten unter der CPAP-Behandlung profitierten wahrscheinlich durch eine Verbesserung der atemmechanischen Be- dingungen. Insbesondere im REM-Schlaf kann der positive endexspiratorische Druck erhöht sein. Da die Lungenüberblähung durch die CPAP-Therapie verringert wird, nimmt die Atemarbeit ab. Einige Studien haben nachgewiesen, dass eine CPAPTherapie die Lungenfunktion, den Gasaustausch und die Atemmuskelfunktion verbessert [3].

Eine Limitation der Studie besteht darin, dass in der Kontrollgruppe Patienten eingeschlossen wurden, die nach drei Monaten eine fehlende Therapieadhärenz zeigten. Es kann deshalb nicht ausgeschlossen werden, dass andere Faktoren zum Überlebensvorteil der mit CPAP behandelten Gruppe beigetragen haben (healthy user effect).

\section{Fazit}

Diese Studie weist auf einen ausgeprägten positiven Effekt einer CPAP-Therapie bei Patienten mit COPD und Tageshypoxämie bei mäßiger bis schwergradiger OSAS hin. Da bei etwa jedem sechsten Patienten mit COPD ein Schlafapnoesyndrom vorliegt, empfiehlt es sich, besonders auf diese Kombination zu achten. Mit einem Fragebogen, der die wichtigsten Symptome erfasst, und einer sich im Verdachtsfall anschließenden nächtlichen Oxymetrie kann die Diagnose mit hoher Wahrscheinlichkeit gestellt werden. Die endgültige Diagnose und eine eventuell erforderliche Therapie sollten unter polysomnografischer Kontrolle mit exakter Titration des Drucks und der Sauerstoff-Flussrate erfolgen.

Bei rechtzeitiger Therapie dürfte es gelingen, nicht nur die Lebensqualität, sondern auch die Lebenserwartung der Patienten zu verbessern.

\section{Literatur}

1. Nocturnal Oxygen Therapy Trial Group. Continuous or nocturnal oxygen therapy in hypoxemic chronic obstructive lung disease. A clinical trial. Ann Intern Med. 1980;93:391-398

2. Report of the Medical Research Council Working Party. Report of long-term domiciliary oxygen therapy in chronic hypoxic cor pulmonale complicating chronic bronchitis and emphysema. Lancet 1981;1:681-685

3. De Miguel J et al. Long-term effects of treatment with nasal continuous positive airway pressure on lung function in patients with overlap syndrome. Sleep Breath 2002;6:3-10 